\title{
Migration saisonnière et maternité : les travailleuses agricoles mexicaines au Canada
}

Seasonal Migration and Maternity: Mexican Women Workers in Canada Migración temporal y maternidad: las trabajadoras agrícolas mexicanas en Canadá

\section{Ofelia Becerril}

\section{(2) OpenEdition}

Journals

\section{Édition électronique}

URL : https://journals.openedition.org/remi/7212

DOI : $10.4000 /$ remi.7212

ISSN : $1777-5418$

Éditeur

Université de Poitiers

\section{Édition imprimée}

Date de publication : 1 janvier 2015

Pagination : 103-120

ISBN : 979-10-90426-24-5

ISSN : 0765-0752

Référence électronique

Ofelia Becerril, « Migration saisonnière et maternité : les travailleuses agricoles mexicaines au Canada », Revue européenne des migrations internationales [En ligne], vol. 31 - $n^{\circ} 1$ | 2015, mis en ligne le 01 janvier 2018, consulté le 14 avril 2022. URL : http://journals.openedition.org/remi/7212 ; DOI : https://doi.org/10.4000/remi.7212 


\section{Note de recherche}

\section{Migration saisonnière et maternité : les travailleuses agricoles mexicaines au Canada}

\section{Ofelia Becerril ${ }^{1}$}

\section{Introduction}

Au cours de cette dernière décennie, les conditions de travail et de vie des travailleurs et travailleuses saisonnier(e)s qui partent depuis le Mexique vers le Canada dans le cadre des programmes SAW et NOC C et $D^{2}$ sont devenues de plus en plus précaires. Les politiques migratoires canadiennes reposent sur une demande croissante de travailleurs temporaires, la privatisation de leur recrutement et la prolifération de formes de contrats incorporant à terme les logiques segmentées de genre, d'appartenance ethnique, de classe et de nationalité. L'évolution de ces politiques a été rapide et a entraîné une " nouvelle ère de migrations saisonnières ". Dès 2002, la présence des travailleurs et travailleuses saisonnier(e)s a augmenté de façon significative et, depuis 2008, leur pourcentage a devancé celui des résidents étrangers permanents (Alboim, 2009 ; Goldring, Hennebry et Preibisch, 2009). Avec la mise en place d'une politique migratoire libérale et l'introduction du « Projet pilote pour les emplois moins qualifiés ", le nombre de travailleurs temporaires est passé de 63441 en 1983 à 213573 en 2012 (CIC, 2008, 2012). En 2002, 2277 travailleurs temporaires ont participé au programme NOC C et $D$, ils étaient 55175 à y prendre part en 2012 (CIC, 2012). Les travailleuses temporaires occupent les emplois moins qualifiés et plus vulnérables (AMSSA, 2013). Elles viennent des États-Unis, qui sont le premier pays fournisseur de main-d'œuvre étrangère, mais également du Mexique (Verea, 2010 : 108-109).

\footnotetext{
1 Docteure en anthropologie. Centre d'études rurales, El Colegio de Michoacán, Martínez de Navarrete 505, Las Fuentes, CP. 59690, Zamora, Michoacán, Mexique ; obecerril@colmich.edu.mx. Article traduit par Catherine Marie Bony Hebert et révisé par Adelina Miranda avec I'autorisation de I'auteure.

2 Les programmes SAW (Seasonal Agricultural Workers) et NOC C and D (National Occupational Classification, Skill Level C or D.) font partie du TFWP (Temporary Foreign Worker Program), programme destiné aux travailleurs étrangers temporaires qui existe depuis 1973. Dès 2002, avec le Project For Occupations Requiring Lower Level of Formal Training, le nombre de travailleurs et travailleuses temporaires au Canada a augmenté.
} 
Les politiques canadiennes ont affecté les migrations mexicaines. En 1998, le Mexique occupait la huitième place parmi les dix principaux pays d'origine des travailleurs temporaires au Canada; en 2012, il se situait au second rang. Avec 9,8 \% de travailleurs, le Mexique se plaçait derrière les États-Unis et devançait la France, le Royaume-Uni, I'Australie, I'Inde, les Philippines, la Jamaïque, I'Allemagne et I'Irlande. Le nombre de Mexicains travaillant sous un statut temporaire au Canada a doublé tout au long de ces dix dernières années, passant de 11630 , en 2003, à 23 683, en 2012. Ces données ne tiennent pas compte des travailleurs du programme SAW. En 2012, les hommes continuaient à représenter la majorité des travailleurs temporaires étrangers : $86 \%$ de 332468 (CIC, 2013). Toutefois, si en 1989 I'on ne dénombrait que 37 femmes, en 2013, sur 17813 travailleurs mexicains, 688 femmes étaient employées dans le cadre du programme SAW (STPS, 2014).

Cet article examine les politiques mises en œuvre dans le cadre du programme SAW par le gouvernement canadien et leurs conséquences sur les mères migrantes mexicaines qui travaillent dans le secteur agroalimentaire ${ }^{3}$. Lorsque ces femmes émigrent dans le cadre de ce programme, leurs familles et foyers d'origine se fragmentent. De ce fait, le processus de précarisation dans la sphère du travail a un fort impact sur la maternité transnationale.

La première partie retrace les cadres théorique et méthodologique utilisés lors de nos recherches ${ }^{4}$; la seconde expose le profil socio-économique des travailleuses mexicaines qui participent au programme $S A W$; la troisième analyse les expériences et les pratiques de ces mères travailleuses au regard des politiques migratoires du gouvernement canadien destinées aux travailleurs agricoles temporaires; la quatrième partie, enfin, examine les politiques migratoires du programme SAW sous l'angle d'une problématique du genre. Nous aborderons principalement la question suivante : comment ces femmes aménagent-elles leur maternité dans le contexte d'une migration inter nationale ? Nous montrerons que ces migrantes saisonnières expérimentent et transforment les pratiques, les relations et les significations attribuées à la maternité pour réorganiser leur travail et leur vie familiale entre le Mexique et le Canada. Ces changements sont multiples tant dans la manière de constituer des familles transnationales que de concevoir la maternité. En effet, l'expérience de la maternité transnationale est hétérogène et dépend notamment du nombre d'années pendant lesquelles les femmes ont travaillé au Canada, de leur statut de mère et d'épouse -avec ou sans conjoint -, du nombre et de l'âge des enfants, et du cycle de vie familiale.

\footnotetext{
3 Pendant plus de deux décennies, le Canada a favorisé l'embauche de mères migrantes (avec ou sans conjoint), chefs de famille, ayant des personnes à charge.

4 Cet article s'appuie sur deux recherches. Une recherche collective intitulée : "Travail transnational, politiques de genre et organisation familiale. Femmes trans-migrantes temporaires du Mexique aux États-Unis et Canada ». Elle a été financée par le Programme inter-institutionnel d'études sur la région d'Amérique du Nord (PIERAN) et du Colegio de México. Nous nous appuyons également sur les résultats d'une recherche personnelle réalisée à Leamington et à Niagara, Ontario, pendant l'été 2009, financée par le Canada-Amérique latine-Caraïbes (CIEC). Ce projet (Stratégies de "gloves-off economy" et conditions de travail de migrants du Michoacán ayant des visas d'emploi temporaire aux États-Unis et au Canada) a fait partie d'un projet plus large financé par le fonds mixte CONACYT et le gouvernement de l'État du Michoacán au Mexique.
} 


\section{Cadres théorique et méthodologique}

Lors de nos recherches, nous avons adopté une approche par genre et une perspective transnationale pour saisir l'expérience vécue par les travailleuses agricoles saisonnières mexicaines qui participent au programme SAW. La référence à ce double cadre conceptuel nous a permis d'examiner les inter actions existant entre sociétés d'arrivée et d'origine et de mettre en avant I'agency, la subjectivité et les stratégies que les femmes migrantes utilisent pour prendre soin de leurs familles.

En effet, la majorité des recherches démontrent que l'appartenance selon le genre façonne les modèles migratoires des hommes et des femmes et leur expérience de vie selon les lieux dans lesquels ils vivent (Hondagneu-Sotelo, 1994). Mais, les dynamiques de genre doivent être aussi analysées en tenant compte du fait que les appartenances de classe, de race et ethniques jouent simultanément leur rôle dans les expériences des migrantes (Pessar, 1999). Cet article utilise cette approche intersectionnelle pour comprendre les modèles migratoires et la conception de l'emploi féminin du programme SAW au Canada ainsi que pour illustrer l'impact que l'emploi temporaire exerce sur les expériences et les dynamiques familiales des travailleuses migrantes.

Notre appareil conceptuel nous a permis de concevoir les pratiques transnationales comme des processus sociaux et de considérer la façon dont les migrantes opèrent dans les domaines de I'action sociale, politique et économique en dépassant les frontières géographiques, politiques et culturelles (Glick Schiller, Basch et Blanc-Szanton, 1992). Notre étude appréhende les circuits migratoires transnationaux comme étant structurés par un mouvement constant de personnes, argent, biens, informations, institutions, idéologies, signes et valeurs qui circulent et unissent les communautés d'origine avec celles créées par les migrants (Rouse, 1989). Ces circuits sont historiquement et géographiquement enracinés dans la réalité mexicaine et ils répondent à des logiques multifactorielles et à des dynamiques locales, régionales, nationales et mondiales (Rivera, 2008).

Dans nos travaux, nous observons l'expérience de travail et la maternité transnationale des femmes migrantes engagées dans des circuits migratoires entre les mondes ruraux du Mexique et du Canada. Pour étudier l'impact des politiques gouvernementales sur le travail et la vie des travailleuses agricoles mexicaines, nous nous référons au cadre conceptuel proposé par Aihwa Ong (1987). Selon cette anthropologue, grâce à leur position changeante à l'intérieur de la famille, du travail et de la société, les femmes peuvent élaborer des stratégies qui leur permettent de résister aux représentations qui leur sont imposées et, en conséquence, de changer la représentation qu'elles ont d'elles-mêmes.

Au cours de notre recherche, nous avons fait un séjour en 2002-2004 et un autre en 2009 à Leamington et Niagara-On-The-Lake en Ontario ; en 2010-2011, nous avons enquêté dans les États de Guanajuato, Michoacán et de Mexico, au Mexique Nous avons utilisé une approche microsociale et une méthodologie mixte qualitative et quantitative (appliquée à la collecte des données, à leur 
analyse et à l'interprétation théorique). Nous avons réalisé des entretiens ${ }^{5}$, retranscrit des histoires de vie et mené un groupe de discussion avec des femmes migrantes; nous avons pratiqué l'observation participante pendant six mois dans les lieux de travail, les lieux de rencontre - tels que les magasins alimentaires - les exploitations et les résidences. Nous avons aussi effectué des entretiens auprès de représentants du secteur agroalimentaire et d'institutions qui gèrent le programme SAW dans les deux pays. Nous avons en outre, interviewé des membres d'organisations qui assurent des services auprès des femmes migrantes : informations sur leurs droits et conditions de travail ; traductions et accompagnement dans les hôpitaux pour les travailleurs blessés ou malades ; suivi de la procédure pour obtenir la sécurité sociale ; préparation de la déclaration d'impôts au Canada ; présentation des demandes d'indemnisation ou d'assurance médicale en cas de rapatriement.

\section{Les travailleuses agricoles mexicaines recrutées dans le cadre du programme $S A W$}

En septembre 2014, 19774 travailleurs mexicains étaient employés au Canada, soit plus du double des 9175 recrutés en 2000 (STPS, 2000, 2014). Si nous regardons l'évolution de cette présence depuis quarante ans, nous constatons qu'ils arrivent désormais de tous les États de la République mexicaine et qu'ils sont présents dans l'ensemble du monde rural canadien, avec une concentration dans dix provinces, et auprès de 1684 employeurs. Les migrants mexicains sont en outre embauchés dans des filières agroalimentaires de plus en plus différenciées et leur temps de présence au Canada s'est allongé. La durée des contrats de travail est plus longue et ils constituent un groupe de travailleurs stable ${ }^{6}$. II s'agit par ailleurs d'une force de travail fortement masculinisé. En effet, à l'origine, seuls les hommes ont pu répondre au programme SAW et l'ouverture de ce programme aux femmes a été très lente. C'est en 1989 que les Mexicaines ont pu se joindre aux hommes. Entre 1989 et 1997, leur nombre est passé de 37 à 67 ; entre 1998 et 2006, de 145 à 395 ; entre 2001 et 2013, de 563 à 688. En 2009, sur un total de 23375 travailleurs du programme SAW, 740 (2,5\% du total) étaient des femmes dont 620 d'origine mexicaine (CIC, 2010 ; STPS 2001-2013). En 2013, les travailleuses mexicaines représentaient 3,7 \% des 18501 travailleurs mexicains de ce programme. En résumé, en vingt-quatre ans (1989-2013), 7566 travailleuses agricoles mexicaines ont été embauchées.

\footnotetext{
5 Nous avons réalisé vingt-huit entretiens avec des travailleuses mexicaines appartenant à différentes communautés d'origine pendant leur séjour à Leamington et Niagara et vingt entretiens avec des travailleuses migrantes lors de leur retour au Mexique. Les entretiens ont été effectués à l'intérieur et à l'extérieur des exploitations agricoles, dans les résidences où vivent les femmes et dans d'autres espaces de rencontre.

6 Le contrat de travail qui est signé entre le migrant mexicain et l'employeur canadien est nominatif. Huit jours après son embauche, le nom du travailleur apparaît sur une liste dite " nominale ". Le contrat établit qu'en cas de rapatriement, le coût total du voyage est pris en charge par l'employeur. Par ailleurs, dans le cas où un " travailleur désigné nominalement " n'est pas disponible pour partir au Canada, I'employeur s'engage à accepter un travailleur substitut (HRSDC, 2012). En réalité, les " travailleurs désignés nominalement " ont déjà travaillé au Canada avec l'employeur qui sollicite leur service pour la saison.
} 
Lors de notre recherche, nous avons pris en considération les travailleuses migrantes qui ont participé au programme SAW entre deux et vingt fois. Nous avons ainsi pu constater une augmentation du nombre des femmes mexicaines participant au Programme et une diversification des origines géographiques. En réponse à l'intensification de la demande de la part des employeurs canadiens, le Mexique a décentralisé le programme SAW et, à partir de 2002, les lieux d'embauche au sein du pays se sont diversifiés. Suivant le modèle général des migrants temporaires mexicains recrutés par le programme, en 2001 les migrantes saisonnières provenaient de treize États ; en 2012, elles arrivaient des trente-trois États de la République mexicaine. La majorité provenait de l'État de Mexico (14,3\%), Tlaxcala (12,2\%), Puebla (10,7 \%), Guanajuato $(8,3 \%)$, Oaxaca $(4,9 \%)$, Veracruz (4,8 \%), Michoacán (4,5\%) et Morelos (4,5\%). Par ailleurs, si en 2000, la grande majorité (91\% du total) des travailleuses mexicaines au Canada était concentrée dans la région d'Ontario, une décennie plus tard, elles étaient embauchées dans cinq provinces : Ontario (39,9\%), Colombie-Britannique $(25,8 \%)$, Alberta $(19,3 \%)$, Québec $(8,8 \%)$ et Saskatchewan $(6,1 \%)^{7}$.

En 2010, sur un total de 621 femmes migrantes employées dans le cadre du programme $S A W, 67,5 \%$ étaient des travailleuses " désignées nominalement " ${ }^{8}$. La majorité d'entre elles comptent désormais entre quatre et quatorze saisons de travail au Canada. Elles sont notamment embauchées pour travailler dans la culture de fruits $(65,2 \%)$, des légumes et fleurs sous serre $(19,8 \%)$, dans les pépinières $(14,4 \%)$, dans les secteurs des primeurs et la production des légumes $\operatorname{secs}(0,6 \%)$. Elles sont employées dans soixante et onze exploitations, principalement à Fraisebec, Tangaro Ventures, Belmor Farms, Meadowbrook Greenhouses, Abe Epp \& Family, Lakeshore, J W Bow Greenhouses, Brar Brothers Farms, Central Botanical Growers, Oyen Greenhouses, Great Northern Hydroponics, et Ricciardelli Orchards, Sun Ray Orchards, Seaway Farms, Rosa Flora, Konkle Farm \& Greenhouses, Andrewes Farms, AVB Greenhouses, et Meyers Fruit Farms (HRSDC, 2010). Dans la municipalité de Niagara-on-The-Lake, quinze exploitations emploient 164 travailleuses et dans la zone de Leamington neuf entreprises en emploient cinquante et une.

Si nous considérons la durée du séjour au Canada, nous pouvons remarquer que la majorité des travailleuses mexicaines arrivent entre janvier et juillet, tandis que les hommes arrivent entre mars et août. En 2006, la majorité (67, 2 \%) des femmes avaient été embauchées pour une moyenne de quatre mois et demi tandis que la grande majorité $(98 \%)$ des hommes avaient reçu des contrats de huit mois (Becerril, 2011). Les femmes migrantes ont donc des contrats de travail plus courts que les hommes, ce qui implique qu'elles gagnent moins et que, en conséquence, elles envoient moins d'argent à leurs familles d'origine. De ce fait, la majorité des femmes passent davantage de temps au Mexique que les hommes et sont obligées de chercher un autre emploi temporaire, généralement dans le secteur domestique ou dans le même secteur agricole.

\footnotetext{
7 En effet, les employeurs canadiens ont choisi de limiter l'embauche des femmes migrantes mexicaines à cinq provinces, situées principalement dans la région de Niagara et de I'aire de Leamington, en Ontario. Les hommes, par contre, peuvent être embauchés dans neuf provinces.
}

8 Sur un total de 15809 hommes migrants, 69,5\% étaient des travailleurs désignés nominalement. 
De plus, bien que les femmes migrantes soient désormais employées dans différents secteurs agroalimentaires, qu'elles effectuent des séjours de travail plus longs qu'auparavant, que la durée de leurs contrats de travail se soit allongée et qu'elles constituent un groupe stable de travailleuses, il n'y a pas eu pour autant une augmentation conséquente de leur salaire. En 2008, par exemple, dans I'Ontario, le taux horaire était de 8,75 dollars canadiens pour travailler dans des exploitations des fruits, légumes, fleurs, arbres de Noël et tabac ; en 2013, il était de 10,25 dollars (HRSDC, 2013). Mais les travailleurs ne se plaignent ni des bas salaires ni des abus dans le paiement par peur d'être rapatriés, licenciés ou que leur nom soit inscrit sur des "listes noires ", ce qui les empêcheraient de revenir travailler au Canada. En 2010, à Simcoe, Ontario, plus de cent travailleurs migrants en provenance du Mexique, de la Jamaïque, de la Trinité et Barbade employés par l'entreprise Ghesquiere ont été rapatriés après avoir fait grève pour exiger les milliers de dollars de salaires non payés (Ramsaroop, 2010). En 2013-2014, d'autres travailleurs migrants ont déposé 242 plaintes contre leurs employeurs notamment pour revendiquer le règlement de leurs salaires et des heures supplémentaires non payées (Hildebrandt, 2014).

Si nous considérons l'âge, le niveau scolaire, le statut matrimonial, le nombre d'enfants et de personnes à charge ${ }^{9}$, nous constatons que le profil des femmes migrantes a changé au cours des dix dernières années. En effet, pour pouvoir participer au programme $S A W$, les femmes doivent vivre en zone rurale, avoir une expérience professionnelle dans le secteur agricole et être âgées de vingt-deux à quarante ans. En 2001, 55 \% des femmes migrantes étaient âgées de dix-huit à trente-quatre ans, mais, en 2010, si $64 \%$ des travailleuses migrantes provenaient encore de zones rurales pauvres du Mexique, leur moyenne d'âge était de trente-huit ans. L'une des raisons de l'augmentation de l'âge de cette population est que certaines travailleuses sont rentrées dans le Programme il y a vingt ans et qu'elles continuent d'y participer. Aujourd'hui, parfois deux générations de mères travaillent dans le cadre du programme $S A W$, comme en témoigne une travailleuse.

"Cela fait dix-sept ans que je travaille au Canada. Ma fille vient aussi au Canada, mais à Alberta. Cela fait quatre ans qu'elle vient travailler dans [la serre] des fleurs.

Elle a trente ans. C'est triste parce que nous ne nous voyons qu'une fois par an ".

(Travailleuse, 51 ans, interviewée à Leamigton, juin 2002)

Un autre changement concerne le niveau scolaire de ces migrantes. Pour candidater, les femmes doivent avoir un bagage scolaire compris entre trois et douze ans. En 2010, 339 (54,6 \% du total) des travailleuses avaient terminé l'école primaire et $266(42,8 \%)$ avaient terminé leurs études secondaires. Mais, si nous analysons le niveau scolaire de ces femmes sur la décennie, nous constatons une augmentation du nombre de travailleuses ayant terminé l'école secondaire : elles étaient 70 sur 280 ( $25 \%$ du total) en 2001 tandis qu'en 2010 elles étaient 266 sur 621 (42,8 \% du total). De plus, en 2010, quatorze de ces femmes $(2,2 \%$ du total) possédaient le baccalauréat. Le chômage et les changements

9 Nous considérons ces variables comme étant déterminantes pour comprendre l'impact des politiques migratoires menées par le gouvernement canadien sur les travailleuses agricoles et leurs familles. 
sociodémographiques du Mexique rural semblent donc favoriser le départ de jeunes mères ayant terminé les études secondaires.

Ces femmes recrutées dans le cadre du programme SAW sont généralement des travailleuses avec enfants. Parmi les migrantes qui ont travaillé au Canada en 2010, 284 sur 621 (45,7\% du total) étaient des mères célibataires, $172(27,7 \%$ du total) étaient séparées de leurs maris, 52 (8,4 \% du total) étaient célibataires sans enfants, $38(6,1 \%$ du total $)$ étaient veuves, $33(5,3 \%$ du total $)$ étaient mariées, 31 (5\% du total) étaient divorcées et 11 (1,8\% du total) vivaient en union libre. Les " mères travailleuses sans conjoint " " " représentaient $84,5 \%$ du total. Ce sont donc les femmes qui vivent dans des foyers monoparentaux qui émigrent. Cette tendance est stable dans le temps. Elle renvoie aux transformations des familles mexicaines qui ont eu lieu tout au long du XXe siècle, en particulier à la suite de l'augmentation des séparations matrimoniales, de la participation des femmes au marché du travail, de l'augmentation du nombre de familles avec des femmes comme chef de famille et de l'émergence de familles matrifocales et transnationales. En 2009, 354 (62,8 \% du total) femmes migrantes sur 564 avaient un ou deux enfants, $171(30,3 \%)$ trois ou quatre enfants et $39(6,9 \%)$ de cinq à huit enfants. En ce qui concerne les hommes migrants, 5773 (45,9\% du total) sur 12583 avaient un ou deux enfants, 5348 (42,5\%) trois ou quatre enfants et $1462(11,6 \%)$ entre cinq et huit enfants. Par ailleurs, entre 2001 et 2009, le nombre de femmes migrantes qui avaient un ou deux enfants est passé à 137 sur 280 (c'est-à-dire 48,9 \% du total), tandis que le nombre de travailleuses célibataires s'est réduit à 50 (17,9\% du total). La probabilité des femmes célibataires d'être embauchées dans le programme a donc diminué progressivement. Nous ne disposons pas de données sur l'âge des enfants, mais nos recherches suggèrent que la probabilité d'émigrer est déterminée, d'une part, par le nombre et l'âge des enfants et, d'autre part, par la présence d'autres femmes appartenant au groupe domestique qui peuvent assumer les fonctions de chef de famille pendant l'absence des travailleuses saisonnières.

En général, les migrantes vivent au Mexique dans des groupes domestiques élargis comprenant des membres adultes qui dépendent économiquement d'elles. En 2009, 99 \% (603 sur 609) de ces femmes avaient à charge entre une et six personnes restées au pays. Les entretiens ont permis de vérifier qu'il s'agissait surtout d'un père ou d'une mère âgés, d'un parent malade, d'un époux ou d'un frère au chômage. La composition des familles d'origine est importante car elle constitue une condition pour pouvoir participer au programme mais également un mécanisme politique qui permet de s'assurer du retour des femmes au Mexique dès la fin de leur contrat au Canada.

\section{Familles et maternité transnationales}

Dans nos recherches, la maternité transnationale renvoie aux " pratiques éducationnelles " que les femmes migrantes mettent en œuvre durant le temps de la séparation d'avec leurs enfants. Dans les localités d'origine, le travail reproductif est principalement confié aux grands-mères. Les arrangements pour les soins des enfants sont négociés simultanément, entre les sociétés d'accueil et d'origine (Parella et Cavalcanti, 2010 ; Pedone, 2010), et ces engagements confèrent une nouvelle signification à la maternité. 
Depuis quelques années, la migration des mères mexicaines employées comme travailleuses agricoles a provoqué des changements multiples et profonds dans les structures et les dynamiques des familles. À partir des résultats de notre travail de terrain, nous proposons une typologie qui tient compte de la manière dont les femmes reconstruisent leur maternité en fonction de leur expérience migratoire transnationale. Nous considérons trois critères. Le premier a trait à l'autoperception, au temps et à la position individuelle que ces femmes migrantes occupent dans la reconfiguration de leur maternité avant, pendant et après leur expérience de travail au Canada. Le second critère concerne I'hétéro-perception, le temps et le cycle de la vie familiale; ce qui permet de regarder la manière dont ces femmes redéfinissent leur rôle à partir de leur statut de pourvoyeuses économiques. Le troisième critère est lié aux contraintes (durée du travail et lieux de travail) relatives à la demande des employeurs qui bouleversent les temps individuels et familiaux des travailleuses saisonnières agricoles. Si nous regardons les différentes variables sociodémographiques qui caractérisent ces mères migrantes, nous pouvons identifier cinq types de maternité transnationale :

- Mères migrantes sans conjoint (célibataires, veuves, divorcées, séparées) définies comme familles monoparentales.

- Mères migrantes avec conjoint (mariées ou en union libre).

- Mères migrantes qui, selon leur statut dans le cadre du Programme, peuvent être " travailleuses sélectionnées " (ainsi dénommées celles qui partent une première fois et qui ne retournent plus au Canada) ou " travailleuses sur liste nominale " qui comptent entre deux et quatorze séjours (et parfois plus) au Canada.

- Mères travailleuses de première ou deuxième génération.

- Mères migrantes qui élèvent des enfants ou dont les enfants dépendent économiquement d'elles.

Les analyses comparatives révèlent la diversité des profils de familles engagées dans la migration (Parreñas, 2005 ; Pedone, 2007 ; Parella, 2007 ; Herrera, 2008 ; Bernhard, Landolt et Goldring, 2009). Chaque famille transnationale développe des stratégies pour assurer l'entretien et le bien-être du groupe domestique (Parella et Cavalcanti, 2010). Même s'ils passent une partie ou la majorité du temps séparés les uns des autres, les différents membres de la famille sont capables de maintenir leurs liens affectifs, de façon plus ou moins stable, d'avoir le sentiment d'appartenir à une même entité et de percevoir, malgré la distance physique, leur bien-être de façon collective (Bryceson et Vuorela, 2002). Ces liens peuvent se conserver, se renforcer ou s'affaiblir, selon les engagements des uns et des autres qui peuvent altérer le sentiment d'appartenance à la famille transnationale (Parella, 2012 ; Skrbis, 2008 ; Herrera, 2005).

Depuis la publication de I'article d'Hondagneu-Sotelo et Avila " I'm here, but I'm there " (1997), de larges débats ont eu lieu portant sur les enfants que les femmes laissent dans le pays d'origine, sur le poids des idéologies de genre dans la stigmatisation des femmes migrantes et de leurs familles, sur la démarche adoptée par ces mères pour reconstruire leurs liens familiaux et enfin 
sur la reconfiguration des pratiques de la maternité dans le contexte migratoire (Herrera, 2008 ; Mummert, 2005 ; Wagner, 2008). Si les approches et les théories qui essaient $d$ 'expliquer la maternité transnationale sont hétérogènes et variées, l'une des plus pertinentes souligne l'impact de la crise de la sphère de la reproduction sociale et l'incapacité des États à garantir les droits économiques et sociaux de leurs citoyens, notamment des femmes et de leurs enfants.

En utilisant ce cadre analytique, nous avons observé que les femmes recrutées dans le cadre du programme SAW mettaient en place des pratiques d'éducation à distance. Elles confient la responsabilité des enfants à d'autres membres féminins de la famille. La situation est cependant particulièrement difficile pour celles qui n'ont pas de conjoint parce que leur décision d'émigrer dépend des engagements qu'elles sont capables de négocier avec d'autres membres de leur famille - habituellement une grand-mère, une tante ou une fille aînée - qui assument la garde des enfants et d'autres responsabilités domestiques pendant qu'elles travaillent à l'étranger.

Au Mexique, toutes les mères migrantes (avec ou sans conjoint) qui veulent participer au programme SAW doivent avoir l'approbation du système national pour le Développement intégral de la famille (DIF); I'objectif de cet organisme étant de renforcer et d'encourager le bien-être des familles mexicaines. Or, on constate que les pères qui émigrent n'ont pas besoin de l'approbation du DIF, puisque I'on préfigure que leurs épouses s'occuperont des enfants. Par contre, les femmes doivent notifier par écrit au DIF le nom de la personne qui sera temporairement responsable de leurs enfants pendant la période où elles travailleront au Canada. Les personnes ayant la garde des enfants doivent remplir certaines conditions : stabilité économique, être de bonne moralité et avoir un emploi " correct ". Pour le DIF, le père est le premier sur la liste familiale des personnes pouvant se voir confier la garde des enfants, suivi par les grandsparents maternels, les grands-parents paternels et les oncles. Étant donné que la majorité des femmes qui participent au programme $S A W$ sont des mères sans conjoint, elles confient en général leurs enfants à leurs mères, sœurs ou filles aînées.

Plusieurs mères interviewées nous disent vivre leur maternité transnationale entre le Canada et le Mexique depuis plus d'une décennie, c'est le cas de cette mère célibataire travaillant dans une exploitation de fraises :

" Je suis mère célibataire.

Ça fait dix ans que je vais travailler au Canada, cinq ans avec des contrats de cinq mois et les autres de sept mois. La première fois que j'y suis allée, mon fils avait cinq ans et maintenant il va en avoir seize.

Mon fils et ma mère dépendent de moi."

(Travailleuse, 38 ans, interviewée à Irapuato, octobre 2009)

La plupart des femmes embauchées dans le cadre du programme SAW sont les seules pourvoyeuses économiques de leur groupe domestique. En 2010, selon le CONEVAL (Conseil national pour l'évaluation de la politique de développement social), $81 \%$ de la population mexicaine vivait sous le seuil de pauvreté. Et, si en 1987, les familles mexicaines pouvaient vivre avec le revenu d'un seul 
membre de la famille, en 2012 le salaire de trois personnes était nécessaire. Les femmes partent donc avec l'espoir d'assurer une vie meilleure à leur famille. Cette considération revient fréquemment dans les entretiens : "Nous sommes ici à cause des hommes, parce qu'ils n'assument pas leurs responsabilités vis-à-vis des enfants".

Être mère célibataire est presque devenu le trait distinctif des migrantes ce qui leur permet de " se ranger sous la même bannière " en cas de problèmes avec le travail ${ }^{10}$.

Ces femmes qui partent sont fréquemment étiquetées comme " mauvaises mères " et sont vues comme celles qui brisent leurs propres familles, coupables de la violence intrafamiliale, et de l'échec scolaire de leurs enfants ainsi que des grossesses de leurs filles adolescentes.

" Je suis mère célibataire. Quand je suis venue au Canada, j'ai laissé mes enfants encore petits, entre cinq et huit ans. Comme personne ne pouvait m'aider, j'ai dû partir... J'ai peur que mon fils - qui est grand maintenant - ne prenne des drogues, qu'il boive, qu'il s'éloigne de moi. C'est ce que je ne veux pas. Quelquefois, je pense que je ne vais plus venir pour une longue période, je vais demander un contrat de cinq mois et pas de

huit, parce que je passe beaucoup de temps ici, loin de mes enfants. Je pense à mes enfants, que je les laisse seuls, je m'éloigne longtemps d'eux et il peut arriver beaucoup de choses dont je peux me repentir. Et je ne veux pas. " (Travailleuse, 42 ans, interviewée à Leamington, juin 2003)

Lorsque ces migrantes rentrent au Mexique, bien souvent leurs enfants les incitent à continuer à aller travailler au Canada.

"Quand je téléphone à mes enfants, ça me réanime [parce que] je sais qu'ils vont bien. Ensuite, je leur dis que je me fatigue beaucoup au travail [et ils me disent] : "Non, maman, t'es partie au Canada, travaille de bon cœur, ne déprime pas,

on t'attend, ne sois pas triste, ne te fais pas de souci pour nous".

On se sent mal, on abandonne ses propres enfants et ici on se sent seule.

[Mais] au retour, les enfants nous attendent les bras ouverts et nous sommes très contentes d'arriver pour les revoir. " (Travailleuse, 26 ans, interviewée à Niagara, juillet 2004)

Durant leur période de travail au Canada (entre quatre et huit mois), les femmes exercent à distance certaines responsabilités : elles donnent des conseils par téléphone, s'occupent des questions de discipline, d'école, de maladie, et contrôlent le bon usage de l'argent envoyé à la personne qui assume la responsabilité des enfants.

"C'est ma dixième période de travail au Canada.

[Le travail ici] signifie faire progresser mes enfants aussi bien économiquement que socialement, ce qui pour moi signifie l'école. Il doit y avoir communication et confiance même si l'un est ici et les autres là-bas. Nous communiquons par téléphone, tous les huit jours.

10 Beaucoup d'employeurs interdisent ou restreignent les visites dans les résidences des travailleuses migrantes. Une grossesse a souvent pour résultat le rapatriement. 
On doit leur expliquer pourquoi on est ici et qu'on attend d'eux qu'ils aillent à l'école. C'est pour ça qu'on est venues, pour qu'ils progressent et pour faire tous les efforts du monde pour eux." (Travailleuse, 28 ans, interviewée à Niagara, juillet 2004)

Une fois que ces mères prennent la décision de travailler au Canada pour garantir un futur meilleur à leurs enfants, certaines doivent négocier leur pouvoir et leur autorité au sein de leur famille. Ainsi cette femme qui a participé au programme SAW pendant cinq ans avec des contrats de travail de huit mois s'explique sur la façon dont elle a tenté de maintenir des rapports affectifs avec ses enfants.

"Ils [ses enfants] s'habituent peu à peu au fait que je ne reviens là-bas [au Mexique] que quatre mois et que je retourne ici. [Mais] le petit m'a dit : "Ne pars plus. J'ai besoin de toi ici, je me sens seul ". Quelquefois, on se pose beaucoup de questions. L'enfant était en sixième année [de primaire] quand [la maîtresse] a fait venir ma mère. Mais elle [ma mère] disait: " J'ai l'impression qu'il ne m'obéit pas ". Quand j'y suis allée [à l'école de son fils au Mexique,] la maîtresse m'a dit : "Je vais vous envoyer chez un psychologue ". [Alors] l'enfant a commencé à avoir un autre comportement. On est arrivés à avoir [une conversation] : "Maintenant je ne suis pas ta maman, je suis seulement ton amie " et à ces moments-là, je ne pouvais pas le gronder. Mais quand je lui disais : "Maintenant je suis ta maman ", alors on bavardait et je [pouvais] lui dire : "Ça c'est pas bien". Ainsi on est arrivés à avoir confiance. Plus tard, lui aussi me demandait : "Dis-moi, comment est ton travail ? Tu parles avec des gens qui ne sont pas mon papa?" [À d'autres occasions] il me disait: " Aujourd'hui je ne suis pas ton fils, je suis ton ami ". [Un autre jour] il me disait : "Aujourd'hui c'est le jour où tu es mon amie parce que je vais te dire quelque chose ". Je l'écoutais, mais je devais rester calme, je devais supporter".

(Travailleuse, 31 ans, interviewée à Niagara, juillet 2009)

La maternité à distance repose sur de longues discussions téléphoniques au cours desquelles les mères essaient de maintenir leur pouvoir et leur autorité, comme le souligne une interviewée :

"Quand j'appelle, je dis quelque chose comme : " Mets ton frère à l'écouteur. Quoi ? II n'est pas là ? Je t'appellerai demain et je veux que vous soyez là tous les deux ! Je veux que vous soyez tous les deux au téléphone!" Et quand je les écoute tous, je sens qu'ils vont bien.". (Travailleuse, 32 ans, interviewée à Leamington, juillet 2009).

Toutefois, certaines mères n'arrivent pas à négocier les changements induits par la distance. L'une des femmes qui a participé à notre recherche nous dit :

"Quelquefois il y a des problèmes là-bas [au Mexique]. On m'appelle au téléphone et ils me disent : "Il est arrivé ceci ou cela " et je me sens impuissante

parce que je ne suis pas là. "

(Travailleuse, 28 ans, interviewée à Niagara, juin 2004)

Les tensions vécues par ces migrantes entre leurs fonctions productive et reproductive sont proportionnelles à la durée de leur absence (Parella et Cavalcanti, 2010). Ainsi, quand elles rentrent au Mexique, elles sont confrontées à de nouvelles difficultés. Le premier dilemme à résoudre concerne la manière de rétablir l'autorité maternelle. Parfois, les enfants n'obéissent plus à leur mère 
ou ils ont de faibles résultats scolaires soit parce qu'ils ne veulent plus étudier soit parce que leur affection s'est déplacée vers la personne qui les garde.

Un second dilemme vécu par les mères concerne la manière dont la séparation temporaire a été vécue par leurs enfants. Ils manifestent des sentiments d'abandon, de solitude, de tristesse ou alors se rebellent contre l'autorité maternelle. De leur côté, ces femmes expérimentent aussi des sentiments d'abandon, d'anxiété, voire parfois une perte d'identité.

"Là-bas, je reste avec eux quatre mois, j'en jouis mais je suis tout le temps envahie par la tristesse, parce que je pense constamment que je dois retourner...Quand j'arrive à l'aéroport, quand je dois retourner...ce sont des pleurs, des pleurs...Une mère est celle qui leur dit: "Mon amour ", celle qui leur donne tout. [Je suis] leur mère par téléphone pendant huit mois et quatre [mois de l'année] celle qui s'occupe d'eux à temps complet.

Je suis une mère "parabole ". Je n'ai rien d'une mère pendant huit mois jusqu'à mon retour et je m'occupe d'eux tout le temps. Il y a toujours eu en moi ce sentiment de culpabilité de ne pas pouvoir m'occuper d'eux toute l'année. Alors, quand je rentre au Mexique, je me consacre complètement à eux. J'arrive et je suis la mère. À partir du moment où je les [mes enfants] laisse avec elle [ma sœur], ils sont sous sa responsablité. "

(Travailleuse, 25 ans, interviewée à Niagara, octobre 2009)

"Ce qui arrive c'est que, quand on retourne au Mexique, on jette à la figure à nos enfants que c'est pour eux qu'on vient travailler au Canada. Quand on arrive, on arrive toute contente avec des cadeaux et tout, mais la magie s'arrête vite parce qu'on commence à voir ou à se rendre compte qu'ils se portent mal. Alors, on se met en colère et on commence même à leur distribuer des fessées, on crie que c'est pour eux qu'on va travailler au Canada, mais ce n'est pas bien ce que nous faisons!" (Travailleuse, 40 ans, interviewée à Niagara, juillet 2004)

Toutefois, et malgré les profondes difficultés à vivre leurs situations, le travail de ces femmes dans le cadre du programme SAW a transformé la vie des membres de nombreuses familles. La mère d'une travailleuse interviewée en témoigne :

" J'ai soixante-huit ans et mon mari aussi est âgé. Nous dépendons tous les deux de notre fille. C'est pour cela qu'elle va travailler au Canada, ça fait cinq ans qu'elle y va." "

(Mère d'une travailleuse, interviewée à Irapuato, octobre 2010)

Si nous considérons que le genre façonne les modèles socioculturels et les expériences migratoires des hommes et des femmes (Hondagneu-Sotelo, 1994), nous devons alors nous interroger sur les raisons pour lesquelles les migrants confient les enfants à leurs épouses tandis que les migrantes ne les confient pas à leurs époux ${ }^{11}$. Pour les mères mexicaines, le paradoxe réside dans le fait que pour maintenir leur famille, elles sont obligées de la quitter (temporairement). Ce " mandat " est généralement imposé aux pères migrants mais on ne l'accorde que rarement aux travailleuses migrantes. Ce manque de reconnaissance découle de la persistance d'une idéologie de genre qui n'identifie les hommes que dans leur rôle de pourvoyeur. Les expériences des travailleurs

11 En 2013, presque 18000 hommes ont participé au programme SAW. 
migrants, pères mexicains, qui participent au programme $S A W$ ressemblent à celles que vivent les travailleurs migrants mexicains aux États-Unis. Les pères que nous avons interviewés ont témoigné de la perte d'autorité et des privilèges patriarcaux dont ils bénéficiaient au Mexique. La majorité a fait allusion aux coûts émotionnels liés à leur absence : ni voir naître, ni voir grandir leurs enfants alors que les épouses, qui ne quittent pas le Mexique, prennent en charge l'éducation des enfants et s'occupent du reste de la famille. Certaines, comme cette interviewée, se lassent des absences prolongées de leurs maris.

"[Je lui ai dit :] tu m'as dit que tu n'allais travailler [au Canada] que pour une période de trois ans, mais cela fait quinze ans que tu y vas.

Ne pars plus, car la prochaine fois tu ne vas pas me trouver." (Épouse d'un travailleur, 36 ans, interviewée dans l'État de Mexico, avril 2011)

\section{Les politiques migratoires canadiennes et l'exploitation de l'appartenance de genre}

Dans cette section, nous analysons le programme SAW sous I'angle des politiques économiques locales, régionales et globales pour explorer les régimes de travail temporaire organisés sur la base du genre, de la race, de la classe, de l'ethnie, de la nationalité et du statut de citoyenneté (Collins, 1995 ; Ong, 1987). De la même façon que d'autres industries hautement compétitives, le secteur agroalimentaire canadien cherche à maximiser les bénéfices en réduisant les coûts du travail moyennant la mobilisation stratégique d'institutions et de politiques fédérales et locales.

Les employeurs du programme SAW utilisent le dispositif de visas accordés dans le cadre du travail temporaire pour segmenter la force de travail suivant l'appartenance de genre, linguistique et culturelle. Certains responsables reconnaissent même que les stratégies d'embauche sont élaborées, d'un côté, pour créer des divisions à l'intérieur de la force de travail et réduire la socialisation entre les travailleurs et, d'un autre côté, pour contrôler les travailleurs et contenir les salaires ${ }^{12}$. La manière dont les entreprises canadiennes productrices de fraises utilisent les travailleuses d'Irapuato, dans l'État de Guanajuato, constitue un bon exemple des stratégies en fonction du genre utilisées par les employeurs du Programme. Ces entreprises les sollicitent pour profiter de l'expérience qu'elles ont acquise au fil des générations dans la culture et la manipulation de la fraise. Cependant malgré leur savoir-faire, ces femmes ne perçoivent pas un salaire de travailleuses qualifiées. En outre, il n'est pas rare que les employeurs demandent aux saisonnières de convaincre d'autres femmes de leur localité d'origine, expérimentées dans le travail de la fraise, de participer au programme. Comme l'a souligné un producteur :

"Les travailleurs mexicains ne sont jamais fatigués et n'ont pas besoin de supervision ; ils savent ce qu'ils ont à faire. " (Employeur, 45 ans, interviewé à Leamington, juin 2003)

12 Pour ces mêmes raisons, les employeurs embauchent des femmes mexicaines pendant une saison et des femmes des Caraïbes pendant une autre, augmentent ou diminuent le nombre de femmes embauchées, en substituant les femmes par des hommes. 
Les employeurs du programme SAW sont dans I'obligation de couvrir les frais concernant les dépenses de logement (sauf en Colombie-Britannique où les travailleurs étrangers temporaires doivent prendre en charge partiellement leurs dépenses) et une partie du prix du billet d'avion aller-retour (coût total en Colombie-Britannique). Ils doivent aussi garantir l'assurance-maladie et enregistrer les travailleurs temporaires au système de sécurité sociale provincial. Toutefois, tous les employeurs ne respectent pas les termes du contrat (nonversement des compensations dues en cas de maladies ou d'accident du travail, non-paiement de l'assurance maladie) ${ }^{13}$. En outre, les salaires ne sont pas toujours versés à temps et les migrants gagnent en général $15 \%$ de moins que les travailleurs canadiens ${ }^{14}$. Mais la situation de chômage qui règne au Mexique entraîne une forte concurrence entre les femmes pour rester dans le programme $S A W$ et les migrantes ne dénoncent pas leurs conditions de travail par crainte de perdre leurs emplois et, bien sûr le principal revenu de leurs familles.

Le Canada a pourtant adopté une législation contre la discrimination au travail et le programme SAW offre tous les outils juridiques (en particulier, en matière de contrats de travail) pour protéger les migrants temporaires, mais ils ne sont presque pas utilisés. Les migrants saisonniers ont beaucoup de difficulté à exercer leurs droits avec leur statut légal de " temporaires " à la différence des travailleurs sous contrat permanent et en premier lieu les Canadiens; ce qui les rend encore plus vulnérables à l'exploitation (Sharma, 2001 ; Gómez, 2011). Les travailleurs migrants en général ne sont pas protégés par les codes du travail canadien. Ils cotisent pour la retraite et paient la prime d'assurancechômage, mais seul un nombre réduit en reçoit les bénéfices car la plupart ne remplissent pas les conditions pour pouvoir les recevoir et leur situation a même récemment empiré. En effet, alors que les travailleurs du programme $S A W$ pouvaient également bénéficier des droits liés à la paternité ou à la maternité s'ils avaient un enfant pendant la période de leur contrat de travail au Canada, en décembre 2012, le gouvernement conservateur du Premier ministre Harper a supprimé les allocations familiales et les congés parentaux destinés aux travailleurs étrangers ${ }^{15}$. Ce changement $n^{\prime}$ est pas neutre notamment pour les mères sous contrat, quand naît un enfant. L'impact des politiques et des pratiques selon le genre du programme SAW est donc notable pour les femmes d'autant que leur possibilité d'accéder aux ressources est plus limitée que celle des hommes alors que leurs contrats sont plus courts. Ces politiques et pratiques discriminatoires ne sont pourtant pas compatibles avec les valeurs établies par la Charte canadienne des droits et libertés. Et on ne peut pas dire que le Canada ait réussi dans son engagement à défendre l'égalité des sexes.

\footnotetext{
13 Les employeurs de la région de Colombie-Britannique n'inscrivent les travailleurs au programme d'assurance maladie qu'après trois mois de travail et au Manitoba I'assurance maladie n'existe que pour les résidents permanents. Par ailleurs, les employeurs ne leur offrent pas de services de traduction et ils ne fournissent pas toujours l'équipement de sécurité ou de protection nécessaire quand les travailleurs utilisent des substances agro-chimiques dangereuses.

14 Ces conditions de travail ont été largement documentées dans différentes recherches (Basok, 2002 ; Binford, 2002 ; Preibisch, 2004 ; Verduzco et Lozano, 2003 ; Becerril, 2007). 15 Ils apportent une contribution estimée à 3,4 millions de dollars au système d'assurance chômage du Canada (Keung, 2012).
} 
Pour Gómez (2011), le refus de l'État de respecter les droits du travail des saisonniers ne constitue pas une négligence involontaire. Pour l'auteur il existe un lien étroit entre les politiques canadiennes qui régissent la migration du travail temporaire et l'objectif de croissance économique.

\section{Conclusion}

Nombre d'observateurs considèrent que le Canada et le Mexique sont face à la nécessité de modifier leur convention bilatérale en matière de migration. Aujourd'hui, beaucoup de travailleurs et travailleuses mexicain(e)s recruté(e) s dans le cadre du programme SAW vivent et travaillent au Canada plus longtemps que dans leur pays d'origine. Ils contribuent à l'économie du pays et à la maximisation des profits dans les secteurs clés en travaillant pour de bas salaires et dans les conditions précaires, que nous avons décrites. Toutefois, de la même façon que leurs homologues masculins, les travailleuses agricoles saisonnières ne peuvent pas solliciter la résidence permanente et, par conséquent, la nationalité. Leur statut de migrant temporaire et la non-éligibilité à la résidence permanente les rendent dépendantes de leurs employeurs qui doivent justifier à chaque saison d'embauche le fait qu'ils ont besoin de leur force de travail afin qu'elles puissent venir travailler au Canada.

Le Mexique a également échoué quant à la protection et la défense des droits de ses ressortissantes en ne sollicitant pas une amélioration de leurs conditions de travail. Cette carence peut être attribuée en partie au fait que le programme est destiné principalement aux travailleurs. Comme l'a affirmé un fonctionnaire du ministère du Travail que nous avons interviewé en 2009 : " s'il n'y a qu'un petit nombre de travailleuses au Programme, il n'est pas important de prêter attention à leur situation ".

\section{Références bibliographiques}

Affiliation of multicultural societies and services agencies (AMSSA) (2013) Temporary Foreign Workers - Part 1 : Statistics and Trends, [en ligne] consulté le 09/04/2014. URL : http://www.amssa.org/files/AMSSA\%20Info\%20Sheet $\% 20$ Issue $\% 2011 \% 20-\% 20$ Temporary $\% 20$ Foreign $\% 20$ Workers $\% 20-\% 20$ Policies $\% 20$ and\%20Analysis.pdf

Alboim Naomi (2009) Adjusting the Balance: Fixing Canada's Economic Immigration Policies, [en ligne] consulté le 16/10/2013. URL: http://www.maytree. com/wp-content/uploads/2009/07/adjustingthebalance-final.pdf

Bakker Isabella and Gill Stephen (2003) Power, Production and Social Reproduction, London et New York, MacMillan-Palgrave, 264 p.

Basok Tanya (2002) Tortillas and Tomatoes. Transmigrant Mexican Harvesters in Canada, Montreal et Kingston, McGill-Queen's University Press, 169 p.

Becerril Ofelia (2007) Transnational Work and Labour Politics of Gender: A Study of Male and Female Mexican Migrant Farm Workers in Canada, in Luin Goldring and Sailaja Krishnamurti Éds., Organizing the Transnational, Vancouver, UBC Press, pp. 228-248. 
Becerril Ofelia (2011) ¡Soy un tunante, cual loco caminante ! Transmigrantes mexicanos a Canadá contendiendo el género, la sexualidad y la identidad, Zamora de Hidalgo, El Colegio de Michoacán, 422 p.

Bernhard Judith, Landolt Patricia and Goldring Luin (2009) Transnationalizing Families: Canadian Immigration Policy and the Spatial Fragmentation of Caregiving among Latin American Newcomers, International Migration, 47 (2), pp. 3-31.

Binford Leigh (2002) Social and Economic Contradictions of Rural Migrant Contract Labor between Tlaxcala, Mexico and Canada, Culture \& Agriculture, $24(2)$, pp. 1-19.

Bryceson Deborah and Vuorela Ulla Éds. (2002) The Transnational Family: New European Frontiers and Global Networks, Oxford et New York, Berg Publishers, $276 \mathrm{p}$.

Citizenship and Immigration Canada (CIC) (2008), Canada Facts and Figures. Immigrant Overview Permanent and Temporary Residents, [en ligne] consulté le 21/12/2013. URL: http://www.cic.gc.ca/english/pdf/research-stats/facts2008.pdf

Citizenship and Immigration Canada (CIC) (2010), Canada Facts and Figures. Immigrant Overview Permanent and Temporary Residents, [en ligne] consulté le 20/11/2013. URL: http://www.cic.gc.ca/english/pdf/research-stats/facts2010.pdf

Citizenship and Immigration Canada (CIC) (2012), Canada Facts and Figures. Immigrant Overview Permanent and Temporary Residents, [en ligne] consulté le 20/11/2013. URL: http://www.cic.gc.ca/english/pdf/research-stats/facts2012.pdf

Citizenship and Immigration Canada (CIC) (2013), Annual Report to Parliament on Immigration, [en ligne] consulté le 23/01/2014. URL: http://www.cic.gc.ca/ english/pdf/pub/annual-report-2013.pdf

Collins Jane (1995) Transnational Labor Process and Gender Relations: Women in Fruit and Vegetable Production in Chile, Brazil and México, Journal of Latin American Anthropology, 1 (1), pp. 178-199.

Ehrenreich Barbara and Hochschild Russel Arlie Éds. (2004) Introduction, in Global Woman. Nannies, Maids and Sex Workers in the New Economy, New York, Henry Holt and Company, pp 21-33.

Glick Schiller Nina, Basch Linda and Blanc-Szanton Cristina Éds. (1992) Transnationalism: A New Analytic Framework for Understanding Migration, toward a Transnational Perspective on Migration, Annals of the New York Academy of Sciences, 645, pp. 1-24.

Goldring Luin, Hennebry Jenna and Preibisch Kerry (2009) Temporary Worker Programs. North America's Second-Class Citizens, [en ligne] consulté le 10/04/2014. URL: http://robarts.info.yorku.ca/files/2012/03/CW_2009_MrO.pdf

Gomez Tatiana (2011) Low Skill Temporary Work and Non-access to Permanent Residence, [en ligne] consulté le 08/11/2011. URL: http://www.focal.ca/images/ stories/Gomez_Labour_Mobility_Low-skill_workers_and_non-access_to_ permanent_residency_e.pdf.

Herrera Gioconda (2005) Mujeres ecuatorianas en las cadenas globales del cuidado, in Gioconda Herrera, Cristina Carrillo y AliciaTorres Éds., Migración ecuatoriana. Redes, transnacionalismo e identidades, Quito, FLACSO, pp. 281-303. 
Herrera Gioconda (2008) Políticas migratorias y familias transnacionales, in Gioconda Herrera y Jacques Ramírez Eds., América Latina migrante : Estado, familias, identidades, Ecuador, Ministerio de Cultura del Ecuador, pp. 71-86.

Hildebrandt Amber (2014) Few Provinces Track Complaints by Temporary foreign Workers. Only Three Provinces Record How Often Vulnerable Employees File Complaints, [en ligne] consultéle 11/08/2014. URL: http://www.cbc.ca/news/canada/ few-provinces-track-complaints-by-temporary-foreign-workers-1.2648734

Hondagneu-Sotelo Pierrette (1994) Gendered Transitions. Mexican Experiences of Immigration, Berkeley, University of California Press, 288 p.

Hondagneu-Sotelo Pierrette (2001) Domestica. Immigrant Workers Cleaning and Caring in the Shadows of Affluence, Berkeley, University of California Press, $318 \mathrm{p}$.

Hondagneu-Sotelo Pierrette and Avila Ernestine (1997) “I'm Here, but I'm There": The Meanings of Latina Transnational Motherhood, Gender and Society, 11 (5), pp. 548-571.

Human Resources and Skills Development Canada (HRSDC) (2010-2012) Temporary Foreign Worker Program, Seasonal Agricultural Worker Program, [en ligne] consulté le 14/10/2013. URL: http://www.esdc.gc.ca/eng/jobs/foreign_ workers/Imo_statistics/annual-agriculture.shtml

Human Resources and Skills Development Canada (HRSDC) (2013) Wages by Commodity, Agricultural Stream and the Seasonal Agricultural Worker Program, [en ligne] consulté le 14/07/2013. URL: http://www.esdc.gc.ca/eng/jobs/foreign_ workers/agriculture/commodities.shtml\#h2.2

Keung Nicholas (2012) Seasonal Migrant Workers Stripped of Parental Benefits, [en ligne] consulté le 19/06/2013. URL: http://www.thestar.com/news/ canada/2012/12/11/seasonal_migrant_workers_stripped_of_parental_benefits. html

Mummert Gail (2005) Transnational Parenting in Mexican Migrant Communities: Redefining Fatherhood, Motherhood and Caregiving, [en ligne] consulté le 09/03/2013. URL: http://www.ciesas.edu.mx/proyectos/mifs2005/papers/03/gail_ mummert.pdf

Ong Aihwa (1987) The Gender and Labor Politics of Postmodernity, Annual Review of Anthropology, 20, pp. 279-309.

Parella Sònia (2007) Los vínculos afectivos y de cuidado en las familias transnacionales. Migrantes ecuatorianos y peruanos en España, Migraciones Internacionales, 4 (2), pp. 151-188.

Parella Sònia (2012) Familia transnacional y redefinición de los roles de género. El caso de la migración boliviana en España, Papers, 97 (3), pp. 661-684.

Parella Sònia y Cavalcanti Leonardo (2010) Dinámicas familiares transnacionales y migración femenina : una exploración del contexto migratorio boliviano en España, in Grupo Interdisciplinario de Investigador@s Migrantes Coord., Familias, niños, niñas, y jóvenes migrantes, Madrid, IEPALA, pp. 93-106.

Parreñas Salazar Rhacel (2005) Children of Global Migration: Transnational Families and Gendered Woes, Redwood City, Stanford University Press, 224 p.

Pedone Claudia (2007) Familias transnacionales ecuatorianas : estrategias productivas y reproductivas, in Víctor Bretón et al. Éds., Ciudadanía y exclusión : Ecuador y España frente al espejo, Madrid, Catarara, pp. 251-278. 
Pedone Claudia (2010) Introducción. Más allá de los estereotipos : desafíos en torno al estudio de las familias migrantes, in Grupo Interdisciplinario de Investigador@s Migrantes Coord., Familias, niños, niñas, y jóvenes migrantes, Madrid, IEPALA, pp. 11-16.

Pessar Patricia (1999) Engendering Migration Studies. The Case of New Immigrants in the United States, American Behavioral Scientist, 42 (3), pp. 577-600.

Preibisch Kerry (2004) Migrant Agricultural Workers and Processes of Social Inclusion in Rural Canada: Encuentros and Desencuentros, Canadian Journal of Latin American and Caribbean Studies, 29 (57-58), pp. 203-239.

Ramsaroop Chris (2010) Migrant Farm Workers Stage Wildcat Strike to Demand Thousands of Dollars in Unpaid Wages: Employer Responds with Deportation, [en ligne] consulté le 07/03/2011. URL : www.justicia4migrantworkers.org/index_ archive.htm

Rivera Liliana (2008) Los trayectos internos e internacionales en la dinámica de formación de çircuitos migratorios transnacionales, in Gioconda Herrera y Jacques Ramírez Éds., América Latina migrante : Estado, familias, identidades, Quito, Ministerio de Cultura del Ecuador, pp. 89-116.

Rouse Roger (1989) Mexican Migration to the United States: Family Relations in the Development of a Trasnational Migrant Circuit, Ph.D. Disertation, Standford University.

Secretaria del trabajo y prevision social (STPS) (2014) Programa de trabajadores agrícolas temporales Mexicanos a Canadá (2000-2014), [en ligne] consulté le 12/05/2014. URL: http://www.stps.gob.mx/bp/secciones/conoce/areas_atencion/ areas_atencion/servicio_empleo/trabajadores_agricolas.html

Sharma Nandita (2001) On Being Not Canadian: The Social Organization of "Migrant Workers" in Canada, Canadian Review of Sociology and Anthropology, 38 (4), pp. 415-439.

Skrbis Zlatko (2008) Transnational Families: Theorising Migration, Emotions and Belonging, Journal of Intercultural Studies, 29 (3), pp. 231-246.

Verduzco Gustavo and Lozano Isabel (2003) Mexican Workers 'Participation in CSAWP and Development Consequences in the Workers' Rural Home Communities, [en ligne] consulté le 12/05/2014. URL : www.colef.mx/sepmig/ wp.../Gustavo-Verduzco-Final-Report-2004.doc

Verea Mónica (2010) Los mexicanos en la política migratoria canadiense, Norteamérica, 5 (1), pp. 93-127.

Vidal Laura et al. (2002) De paraíso a Carolina del Norte. Redes de apoyo y percepciones de la migración a Estados Unidos de mujeres tabasqueñas, Migraciones Internacionales, 1 (2), pp. 29-62.

Wagner Heike (2008) Maternidad transnacional : discursos, estereotipos y prácticas, in Gioconda Herrera y Jacques Ramírez Éds., América Latina migrante : Estado, familias, identidades, Quito, Ministerio de Cultura del Ecuador, pp. 325-340. 